\title{
From Pathways to People: Applying the Adverse Outcome Pathway (AOP) for Skin Sensitization to Risk Assessment
}

\author{
Cameron MacKay, Michael Davies, Vicki Summerfield, and Gavin Maxwell \\ Safety \& Environmental Assurance Centre, Unilever, Colworth Science Park, Sharnbrook, Bedford, UK
}

\begin{abstract}
Summary
Consumer safety risk assessment of skin sensitization requires information on both consumer exposure to the ingredient through product use and the hazardous properties of the ingredient. Significant progress has been made in determining the hazard potential of ingredients without animal testing. However, hazard identification is insufficient for risk assessment, and an understanding of the dose-response is needed. Obtaining such knowledge without animal testing is challenging and requires applying available mechanistic knowledge to both assay development and the integration of these data. The recent OECD

report "The Adverse Outcome Pathway for Skin Sensitization Initiated by Covalent Binding to Proteins" presents the available mechanistic knowledge of the sensitization response within an adverse outcome pathway (AOP). We propose to use this $A O P$ as the mechanistic basis for physiologicallyand mechanistically-based toxicokinetic-toxicodynamic models of the sensitization response. The approach would be informed by non-animal data, provide predictions of the dose-response required for risk assessment, and would be evaluated against human clinical data.
\end{abstract}

Keywords: adverse outcome pathway, skin sensitization, toxicokinetics, toxicodynamics, models

\section{Background}

Determining the risk of acquiring skin sensitization, and its subsequent clinical manifestation as allergic contact dermatitis (ACD), is a crucial aspect of assuring consumer safety for a wide variety of home and personal care products. Decisions on consumer safety are based on a risk assessment of the product ingredients that takes into account both the hazardous properties of the ingredients and the consumer exposure scenario specific to the product. When skin exposure is expected, an understanding of the hazardous property of the ingredient with respect to skin sensitization is sought, and for this purpose the mouse local lymph node assay often is regarded as the in vivo assay of choice (Basketter et al., 2002; Felter et al., 2002, 2003). The utility of the mouse local lymph node assay lies not only in hazard identification (the ability to identify potentially sensitizing ingredients) but also as a hazard characterization assay, i.e., with the ability to determine a dose-response relationship and a definable potency. The ability to make risk assessment decisions without animal testing will rely upon the confident prediction of this dose-response relationship in humans.

Both the biology and chemistry of skin sensitization have been well studied over a number of decades, and a clear picture of the key steps in the mechanism of action is emerging (Basketter and Kimber, 2010; Lepoittevin et al., 2011). This mechanis- tic knowledge was reviewed recently in the OECD report, "The Adverse Outcome Pathway for Skin Sensitization Initiated by Covalent Binding to Proteins" (OECD, 2012a) and distilled into a number of key events: penetration of the sensitizing ingredient into the viable layers of the skin; modification of skin protein by the sensitizing ingredient (either directly or via metabolic or abiotic transformation of a precursor); production of danger signals and inflammatory mediators by keratinocytes, fibroblasts, and skin-resident dendritic cells; uptake, processing, and presentation of modified protein by dendritic cells; maturation and migration of dendritic cells to the local lymph node; antigen presentation to specific naïve $\mathrm{T}$ cells, and subsequent $\mathrm{T}$ cell proliferation and differentiation; and the generation of a sufficient population of antigen-specific memory $\mathrm{T}$ cells required to mediate an elicitation response at the site of re-exposure. In humans, the resulting elicitation response is observed clinically as an eczematous skin reaction.

This mechanistic knowledge has motivated the development of a number of methods for assessing chemical sensitization hazard potential or potency without the need for animal testing (Adler et al., 2011). Expert weight of evidence approaches for interpreting the combined results from these test methods have shown that it is now increasingly feasible to identify potential skin sensitizing ingredients without the use of animal data (Bauch et al., 2012). Additionally, data-driven approaches

Received January 25, 2013; accepted in revised form April 24, 2013; Epub June 7, 2013 
for predicting sensitizer potency also have been developed (Jaworska et al., 2011, 2013). As of yet, no attempt has been made to predict the human dose-response relationships required for consumer safety risk assessment. Moving from a single in vivo assay to such a robust interpretation of a number of in vitro, in chemico, and in silico methods, each assessing a key event, requires both a significant level of mechanistic insight as well as mechanistically-defensible methods with which to integrate disparate data types (Jowsey et al., 2006).

The OECD report presents an adverse outcome pathway (AOP) for skin sensitization (see Fig. 1). An AOP has been defined as "a conceptual construct that portrays existing knowledge concerning the linkage between a direct molecular initiating event and an adverse outcome at a biological level of organization relevant to risk assessment" (Ankley et al., 2010). The molecular initiating event (MIE) and the adverse outcome serve as anchors at each end of the AOP, with the intermediate relationships across levels of biological scale provided by available mechanistic, causal, or associative information from relevant in vivo, in vitro, or computational approaches. As AOPs are based on available mechanistic knowledge they can be highly detailed or sparsely populated, depending on the level of information available and required by the risk assessment problem. By formalizing this knowl- edge, an AOP can offer a sound mechanistic basis for a transparent risk assessment. However, a methodology for quantification is key: quantification of the resulting dose-response and response-response relationships across the specified levels of biological organization would be required to judge the likelihood of an adverse response occurring for a given ingredient exposure (OECD, 2011).

AOPs detail the known molecular, biochemical, cellular, organ- and organism-level effects of a toxicant on the biological system. As such, quantification would enable the mapping of the magnitude of the MIE to the likelihood and/or severity of the adverse outcome, i.e., provide the toxicodynamics (TD) of the response. Correspondingly, quantification of how consumer exposure determines the ingredient concentration at the site of the MIE provides the ingredient toxicokinetics (TK). Taken together, these quantitative descriptions of the toxicokinetics and toxicodynamics provide the relationship between consumer exposure and the likelihood and/or severity of the adverse outcome: this is the information required in order to determine a safe exposure. Mathematical and statistical modeling has long been used in this context to interpret experimental results from animal-based TK and TD studies (Andersen, 2003; USEPA, 2005; Thompson et al., 2008). More recently, however, efforts have turned to building a priori predictive TKTD models that

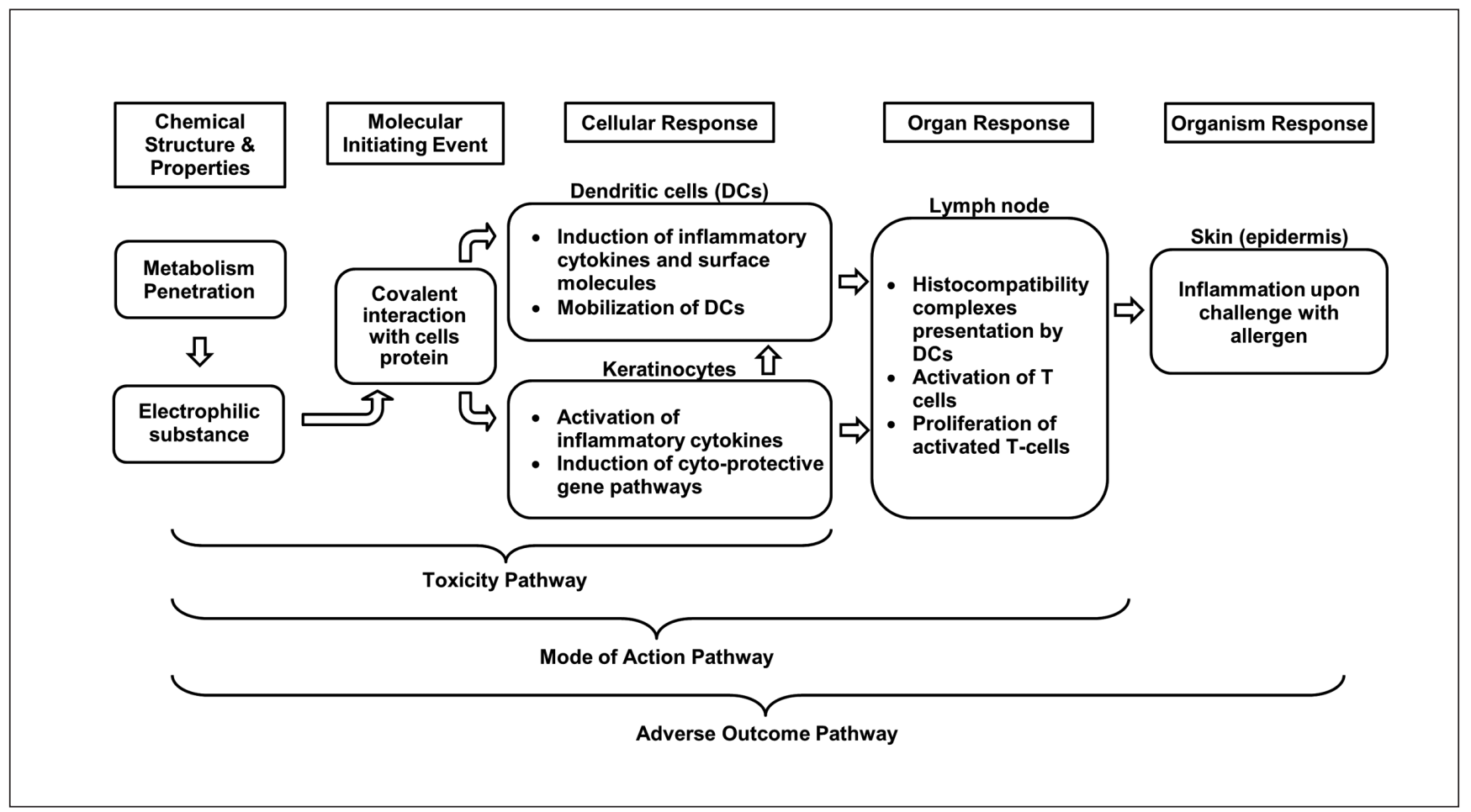

Fig. 1: Flow diagram of the pathways associated with skin sensitization

Covalent modification of skin proteins (haptenation) is identified as the molecular initiating event and the inflammation response in skin observed clinically as allergic contact dermatitis as the adverse outcome. Of the intermediate steps, a further three causal events were identified: biochemical signaling in keratinocytes, activation and mobilization of dendritic cells (DC), and activation and proliferation of T cells in the lymph node. Reproduced with permission from "The Adverse Outcome Pathway for Skin Sensitization Initiated by Covalent Binding to Proteins - Part 1 Series on Testing and Assessment No.168." http://search.oecd.org/officialdocuments/ displaydocumentpdf/?cote=env/jm/mono(2012)10/part1\&doclanguage=en (OECD, 2012a, Fig. 3). 
exploit knowledge of the ingredient physicochemistry, in vitro data, species-specific physiology, and the molecular and cellular mechanisms of toxicity (Shuey et al., 1995; Lau et al., 2000; Maxwell and Mackay, 2008; Bhattacharya et al., 2010, 2011; Louisse et al., 2010; Yoon et al., 2012). These approaches also are being investigated in the context of interpreting highthroughput in vitro toxicity screening data and sublethal effects in environmental risk assessment (Rotroff et al., 2010; Ashauer et al., 2011; Judson et al., 2011; Watanabe et al., 2011). Here, we propose an approach to risk assessment of skin sensitization without animal testing using tailored data to support physiologically- and mechanistically-based toxicokinetic-toxicodynamic (TKTD) prediction models. A requirement of these models is that we advance both our qualitative and quantitative understanding of the skin sensitization response in humans at the molecular, cellular, and clinical levels. We consider the consumer exposure and skin ADME properties as the mechanistic basis for toxicokinetic modeling and the OECD AOP for skin sensitization as the mechanistic basis for toxicodynamic modeling. We outline the practical first steps in such an approach to consumer safety risk assessment and highlight the scientific and technical challenges likely to be faced.

\section{Toxicokinetics of skin allergy}

Toxicokinetics often is referred to as "what the body does to the ingredient" in terms of absorption, distribution, metabolism, and excretion (ADME). TK models can explicitly and/or implicitly model these processes to predict an internal dose metric: ideally, the free concentration of the ingredient at the target or targets that initiate the toxicological mechanism of action. Historically, toxicokinetic models have been derived for systemic exposure estimates by using an empirical, model-fitting approach based on toxicokinetic data generated in animals. Ordinary differential equations (ODE) are used to represent biological compartments, including a central compartment representing blood plasma (Welling, 1997; Boroujerdi, 2001). More recently, physiologically-based approaches have been developed that explicitly represent multiple organs with the required parameters, such as gut absorption rates, blood-organ partitioning, and hepatic clearance rates, predicted either a priori from chemical structure or in vitro data, or determined by fitting the models to blood plasma concentration-time profiles (Reddy et al., 2005). The utility of such models has been in extrapolating across species, dose level, and route of exposure (Thompson et al., 2008). We discuss how these TK approaches can be applied to the ADME processes in skin to predict local skin concentrations following topical application.

Within the AOP framework, the ingredient interaction with the target that initiates the mechanism of action is referred to as the MIE. As was highlighted in the OECD report, the targets for skin sensitization are understood to be proteins in the skin, and the MIE is understood to be the covalent modification of their nucleophilic residues (Landsteiner and Di Somma, 1938; Basketter et al., 1995; Lepoittevin et al., 2011). This process is known as haptenation, and the sensitizing ingredient desig- nated a hapten, pro-hapten, or pre-hapten depending on whether it is directly acting or indirectly acting via abiotic or metabolic transformation. Thus, ideally, the internal dose metric for a toxicokinetic model for skin sensitization would be the free concentration of the ingredient at the resident site of the target proteins. In general, whether, these target proteins reside predominantly in the intra- or extracellular space or on the membrane of a specific cell type is unknown. However, the epidermis and dermis generally are regarded as the skin sites where these modifications become available to the immune system through epidermal Langerhans cell (LC) and dermal dendritic cell (dDC) uptake and migration (Kimber and Dearman, 2003; Pickard et al., 2009; Kimber et al., 2011). In the absence of specific knowledge of the location of target proteins and how it varies across sensitizing ingredients, a practical starting hypothesis for the dose metric would be the free concentration of sensitizing ingredient in the viable skin (Basketter et al., 2007). The TK modeling task, then, is one of predicting how this concentration varies over time following a defined exposure to the ingredient on the surface of the skin.

Before discussing the toxicokinetic events in skin we highlight the importance of the product use scenario in determining the actual exposure experienced by the consumer to the ingredient of concern. The product format (e.g., shampoo, skin cream, deodorant), frequency of use, and the actual ingredient concentration within the product all contribute to determining the actual concentration of ingredient applied to the skin within a given timeframe (see "Product Use" in Fig. 2) (Api et al., 2008). With the amount of ingredient delivered onto the skin at a given concentration defined, a number of toxicokinetic processes occur that ultimately could affect the free concentration of ingredient at the target site (see "Toxicokinetic Events in Skin" in Fig. 2). The significance of these processes in determining free concentration at the target site will vary depending on the chemical properties of both the ingredient and product formulation. The most immediate events are likely to be interaction with the product formulation and evaporation from the formulation. Other abiotic transformation processes, such as autoxidation, also are likely to take place in the product formulation, either rendering the transformed ingredient's sensitization potential inert (clearance), or, in the case of pre-haptens, possibly activating it (Karlberg et al., 2008; Christensson et al., 2010). The route of entry into the viable skin is via passive diffusion through the stratum corneum. Depending on lipophilicity and molecular weight, the rate and route of transport across stratum corneum varies, predominating through either the lipid bilayers, corneocytes or via appendages such as sweat ducts and hair follicles (Potts and Guy, 1992; Mitragotri, 2003; Magnusson et al., 2004). Once present in the viable skin, the ingredient will distribute between extra- and intra-cellular space, where there is the potential for both phase I (activation of pro-haptens) and phase II (predominantly clearing) metabolism (Gibbs et al., 2007; Hagvall et al., 2008; van Eijl et al., 2012). Finally, the ingredient is cleared from the viable skin via the dermal capillary bed.

To determine the free ingredient concentration in the viable skin following topical application and how it varies over the 


$\begin{array}{cccc}\begin{array}{c}\text { Concentration } \\ \text { in product }\end{array} & \begin{array}{c}\text { Concentration } \\ \text { in organism }\end{array} & \text { Concentration } & \text { Molecular } \\ \text { Initiating Event }\end{array}$

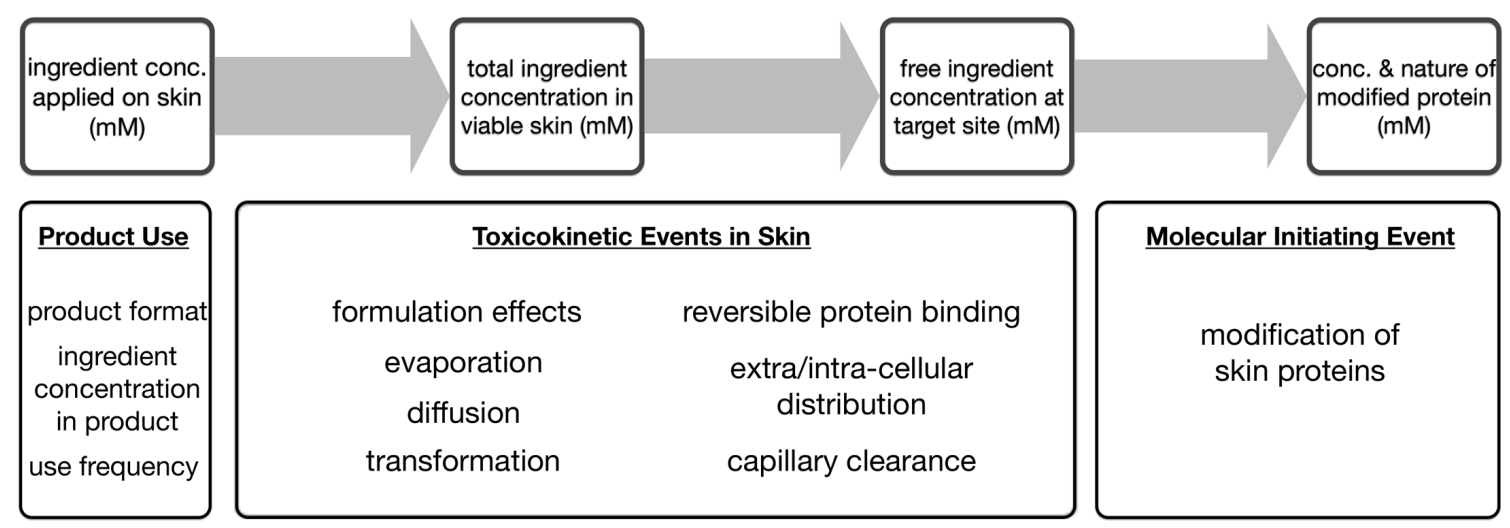

Fig. 2: Relating the concentration at the site of exposure to that at the target site of the molecular initiating event as defined by the AOP for skin sensitization

The key metrics (variables) of sensitizing ingredient exposure across levels of biological organization: from concentration in the product to that at the target site. The "Product Use" and key "Toxicokinetic Events in Skin" are explicitly considered in relating the TK model input, concentration applied on the skin $(\mathrm{mM})$, to the output, free concentration at the target site $(\mathrm{mM})$. This is the concentration available to drive the molecular initiating event, i.e., the modification of skin proteins.

range of chemical properties relevant to consumer products, an understanding of the relative rates of the above skin toxicokinetic events and processes is required. When these rates can be estimated with acceptable accuracy and precision, the effect of these processes on the free concentration of ingredient in the viable skin could be determined and compared within a toxicokinetic model (see Fig. 3). A number of modeling approaches have been applied to understanding the toxicokinetics of skin and have been reviewed extensively elsewhere (Mitragotri et al., 2011). Early work on skin focused on understanding to what extent an ingredient would be systemically available following dermal exposure, leading to a number of data-driven quantitative structure-permeation relationship (QSPR) models that make predictions of ingredient flux across skin based on ingredient lipophilicity and molecular weight (Potts and Guy, 1992). However, more recently, physiologically-based compartmental models of varying complexity have been investigated from both a theoretical and experimental standpoint, and where the TK of local skin concentrations are considered explicitly (McCarley and Bunge, 2001; Pendlington et al., 2008). In this approach, the variables of the model represent the ingredient concentration in the major skin compartments, and the model parameters (diffusion and partitioning) are determined by fitting to time course data on ingredient concentration in the compartments (Davies et al., 2011). This data-driven modeling approach provides a description of the free concentration of ingredient in the viable skin over time and enables extrapolation to repeat dose scenarios and other doses to those originally investigated experimentally. However, the in vitro nature of the experimental approach severely restricts the model utility, as a number of processes, such as metabolic transformation and capillary clearance, are expected to differ substantially from what would likely be observed in vivo.

More in vivo relevant modeling approaches have been investigated with a model structure that takes into account the majority of the key toxicokinetic processes discussed, such as evaporation, diffusion, reversible protein binding, and capillary clearance, and to make a prediction of the free concentration in viable skin over time (Ibrahim et al., 2012; Dancik et al., 2013). The model structure at the macro-scale is similar to the compartmental model approach, with formulation, stratum corneum, and viable tissues treated as separate compartments but with a partial differential equation (PDE) approach used to track ingredient concentration as a continuous function of skin depth. This continuum PDE approach is of particular importance for describing ingredient transport across compartments with high spatial heterogeneity, such as the stratum corneum. Parameterization of the required macro diffusion rates can be achieved by fitting to clinical data encompassing tape stripping, TEWL, or Raman confocal microscopy measurements (Herkenne et al., 2008; Bonnist et al., 2011). However, through consideration of the structure and hydrophobicity of lipid bilayers and corneocytes, significant effort has been made to determine the required diffusion and partition coefficients using only physicochemical 


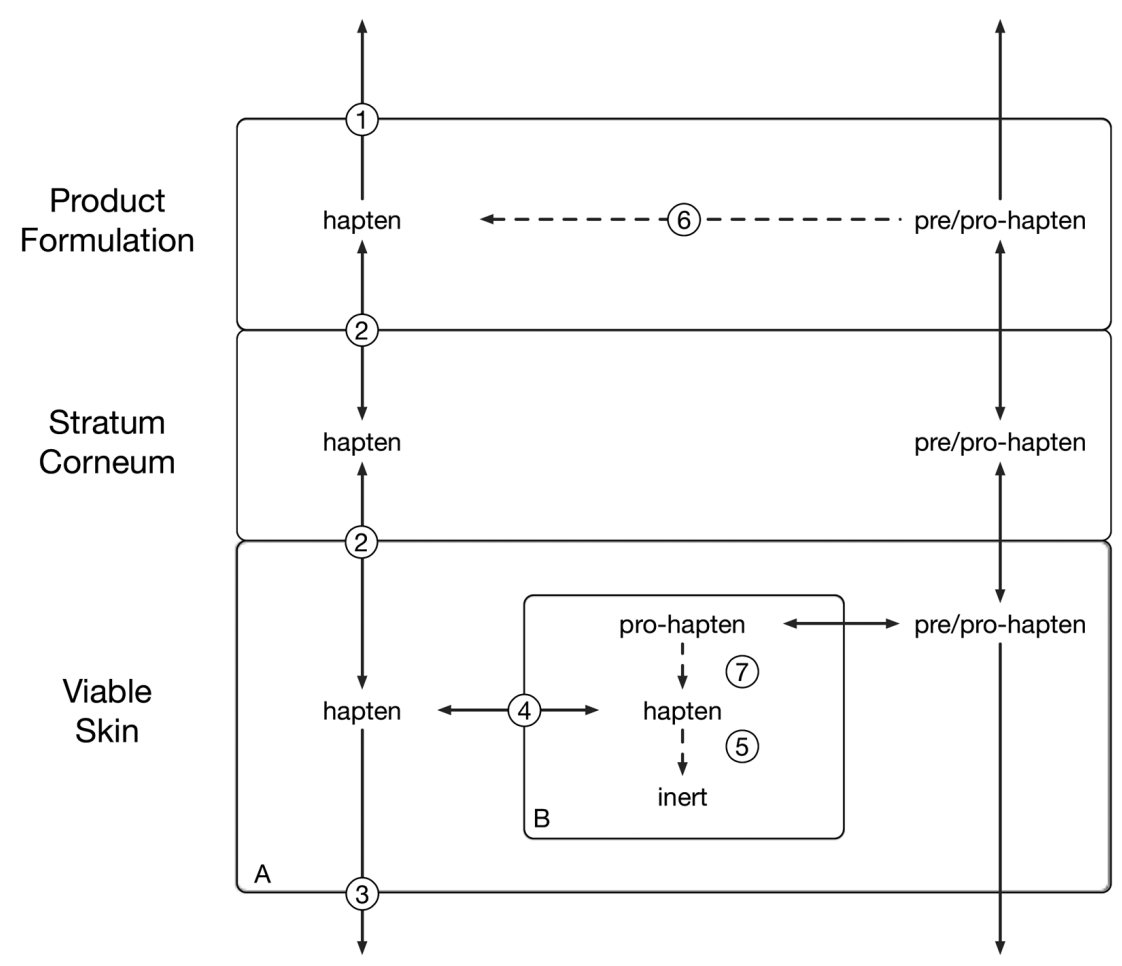

Fig. 3: Example structure of a physiologically-based toxicokinetic model for skin sensitization

Locations that might be considered required for a physiologically-based toxicokinetic model for skin sensitization: concentration in product formulation; concentration in stratum corneum; ingredient (hapten, pro-hapten, or pre-hapten) concentration in the A) extracellular and B) intracellular space of viable skin. Processes that would likely be represented: 1) evaporation from product formulation; 2) diffusion across skin layers; 3 ) capillary clearance; 4) extra/intra-cellular distribution; 5) clearance of the ingredient by transformation (e.g., Phase II metabolism); 6) pre-hapten activation by abiotic transformation; 7) pro-hapten activation by enzymatic transformation (e.g., Phase I metabolism).

descriptors such as molecular weight and $\log \mathrm{P}$ (Wang et al., 2006). Nevertheless, these sub-models still require a level of parameter fitting against data that limits their applicability in providing the required parameter estimates in unexplored regions of chemical space. To make further progress will require considerations at the micro-scale and, to that end, molecular dynamics simulations have been applied to determine both the microscopic diffusion coefficient in SC lipid bilayers and the rate of keratin binding in corneocytes (Rim et al., 2009; Notman and Anwar, 2013). Although computationally expensive, these simulation results can be used to generate the bulk macro-scale parameters required by the continuum-based PDE approaches.

Significant progress has been made in skin toxicokinetic modeling, but some key challenges remain. Transformation of the ingredient in formulation and skin are thought to be of significance in skin allergic response. In formulation, the process is likely to be abiotic transformation via autoxidation and hydrolysis, but in the viable tissues of the skin, enzyme- driven transformation is likely. Although these processes have been largely ignored in current skin toxicokinetic models, addition of model structure to accommodate metabolic clearance and activation is less of a challenge than the experimental approaches that would be needed for parameterization of such models (Jacques et al., 2010; Zalko et al., 2011; Götz et al., 2012; van Eijl et al., 2012). Additionally, the effects of product formulation on ingredient partitioning and diffusion through skin are thought to be of significance and are not well understood. Aspects of cosmetic formulation such as the water/oil or oil/water basis of the emulsion, the surfactant organization, and other structural and chemical properties are likely to affect the skin toxicokinetics of sensitizing ingredients (Otto et al., 2009). An understanding of these effects is required for accurate prediction of subsequent skin toxicokinetics under a variety of product formats.

Ultimately, the evaluation of these models with relevant in vivo data is technically challenging. A number of approaches, such as tape stripping and Raman spectroscopy, can be used to determine ingredient concentration in the upper layers of the stratum corneum and the epidermis, but deeper layers are only accessible via disruptive procedures such as micro-dialysis and punch biopsies (Holmgaard et al., 2010; Bonnist et al., 2011; Wang and Maibach, 2011). Evaluation of such models over a wide range of ingredient chemistries and formulations is a challenging prospect. Addressing these challenges is key to developing a quantitative understanding of skin toxicokinet- 
A

Toxicokinetic Model

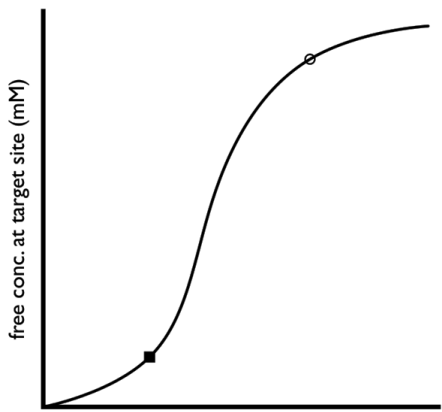

conc. applied on skin (mM)

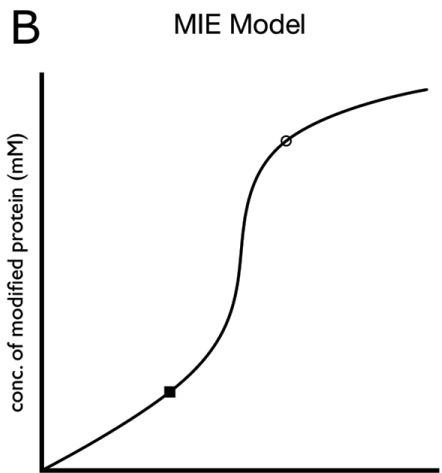

free conc. at target $(\mathrm{mM})$
C Toxicodynamic Model

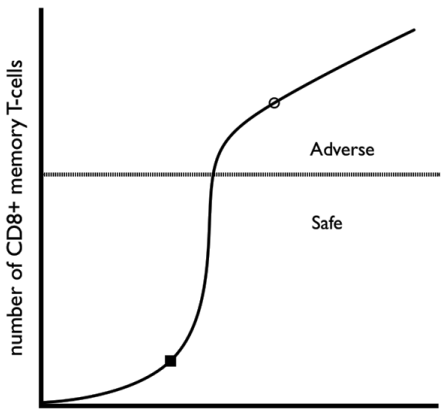

conc. of modified protein $(\mathrm{mM})$

Fig. 4: Quantifying the relationship between exposure and response

A) Relationship between external dose and dose at target as provided by a mechanistically-based toxicokinetic model for skin sensitization. B) Relationship between dose at target and MIE activation as provided by a model of the binding kinetics of the sensitizing ingredient. C) Relationship between MIE activation and the adverse response as provided by a mechanistically-based toxicodynamic model of the antigen-specific $T$ cell response. Linking inputs and outputs across models would provide the exposure-response relationship and the likelihood of whether the exposure is adverse or safe. Graphs are intended to be illustrative and do not represent real data or model output.

ics. However, drawing on predictions from the available skin toxicokinetic modeling approaches discussed above would represent a practical first step in determining the free concentration of the ingredient at the target site (see Fig. 4A).

\section{The molecular initiating event}

Physiologically-based toxicokinetic models of skin can provide the ability to predict the free concentration at the site of the MIE following a defined topical exposure, but relating this to the likelihood of an adverse outcome requires an understanding of both the nature and magnitude of the MIE and the subsequent toxicodynamics of the response. The MIE is the interaction between a chemical and a biological target (generally thought of as molecular) sufficient to cause subsequent events at higher levels of biological scale (e.g., cellular, organ) and ultimately culminating in the adverse response as detailed by an AOP (Ankley et al., 2010). The MIE for skin sensitization considered in the OECD AOP and widely accepted as the key causative event is haptenation: the modification of skin protein by a sensitizing agent, the hapten (Lepoittevin et al., 2011; OECD, 2012a) (see "Molecular Initiating Event" in Fig. 2).

Sensitizers by themselves are not large enough to be recognized by the immune system and so the importance of haptenation is that it provides a source of antigen in the form of modified protein, which the immune system recognizes as nonself (Martin and Weltzien, 1994; Weltzien et al., 1996). The modification of proteins by sensitizers generally is regarded as an irreversible reaction with the rate of modification dependent on the free concentration of the sensitizing ingredient, the free concentration of protein or peptide, and the inherent rate of the reaction. Given the importance of this step to the adverse response, a significant amount of experimental and theoretical chemistry work has been devoted to determining the reactivity of sensitizing ingredients and the reaction rates of ingredients with model proteins and peptides (Böhme et al., 2009; Roberts and Natsch, 2009; Chipinda et al., 2010). However, the simple experimental systems used to determine reaction kinetics will differ from that of skin in a number of ways, such as: competition for binding between the mixture of proteins present, difference in protein expression levels, difference in $\mathrm{pH}$ and steric hindrance. As such, it remains an open question as to how representative chemical kinetics derived from experimental measurements in simple systems might be of the those occurring in viable skin. Some recent, and perhaps more biologically relevant, experimental studies have investigated sensitizer exposure to keratinocytes in vitro have been able to identify the sites of protein modification and thus peptides that may be immunogenic (Bauer et al., 2011; Simonsson et al., 2011).

We are far from having models that can predict the concentration and specificity of sensitizer-induced protein modifications in skin. Nevertheless, the free ingredient concentration in skin (from the TK modeling approaches discussed above) and an experimentally derived ingredient-protein reaction rate could be used to estimate a bulk concentration of modified protein in skin 
using the appropriate chemical kinetics model. Estimating the bulk concentration of modified protein in skin may be regarded as a practical first step in developing a better quantitative understanding of the magnitude of the MIE for a given topical exposure (see Fig. 4B).

\section{Toxicodynamics of skin allergy}

A combination of physiologically-based toxicokinetic models of skin and chemical kinetics modeling may provide an estimate of bulk concentration of modified protein in skin. However, relating this to the likelihood of an adverse outcome requires the addition of a toxicodynamic modeling approach. Toxicodynamics is often referred to as "what the ingredient does to the body" initiated by the ingredient's toxicological mechanism of action via the MIE. Here we use the term toxicodynamic model to encompass any mathematical modeling approach that utilizes the available mechanistic knowledge for determining the relationship between the magnitude of the MIE and the likelihood or severity of the adverse response. Such a broad definition also encompasses the "toxicity pathways" modeling proposed by the NRC report "Toxicity Testing in the $21^{\text {st }}$ Century" but has the explicit modeling aim to include the necessary detail across biological scale to enable prediction of the likelihood or severity of the adverse response (Krewski et al., 2010; Bhattacharya et al., 2011).

The OECD AOP for skin sensitization highlights the successful presentation of modified peptides resulting in antigen-specific $\mathrm{T}$ cell proliferation and the formation of a pool of effector and memory $\mathrm{T}$ cells and their mediation of the elicitation response as a key causal event. This results from a number of coordinated toxicodynamic events (see "Toxicodynamic Events of the AOP" in Fig. 5). Initial $\mathrm{T}$ cell contact with the immune system occurs in the lymph node where the immigrant antigen presenting cells (APCs) present processed haptenated protein (the antigen) to the resident $\mathrm{T}$ cells. It is not known how haptenated protein is processed by APCs (Langerhans Cells and dermal dendritic cells), however the involvement of $\mathrm{CD}^{+}$and $\mathrm{CD}^{+} \mathrm{T}$ cells in the response is suggestive of presentation via both major histocompatibility complex type I (MHCI) and II (MHCII). Sensitizer-derived antigenic peptide presented on MHC (pMHC) could be generated by a number of different routes: processing of protein haptenated outside the APC; processing of protein haptenated inside the APC; cross presentation of peptide loaded MHC from another cell; or by direct haptenation of peptides already presented on the MHC. Antigen presentation to specific naïve $\mathrm{T}$ cells causes clonal expansion and the generation of an antigen-specific memory $\mathrm{T}$ cell population. The amount of expansion from a naïve $T$ cell is dependent on the duration of the synaptic interaction between the APC and the T cell. This, in turn, is determined by the affinity of the pMHC for the $\mathrm{T}$ cell receptor (TCR), the amount of pMHC on each APC, and the level of co-stimulatory molecule expression (see "Cellular Response" in Fig. 5).

The ability to measure and predict these three variables from existing knowledge and experimental techniques is limited. The importance of pMHC affinity for TCR is recognized but the exact nature of how binding affects activation is debated (Carreño et al., 2006; Stone et al., 2009; Morris and Allen, 2012). The affinity profile of presented pMHCs for a given TCR depends on the nature of the pMHCs itself. Understanding this requires knowledge of the structural nature of the protein modifications in skin. Additionally, the amount of a specific modified pMHC on an APC depends on the likelihood of source modifications being presented by APCs. Experimental studies have been performed where peptides have been eluted from cells and sequencing performed by mass spectrometry to gain an understanding of the self-peptide repertoire that can be presented by MHC (Hickman et al., 2004; Gebreselassie et al., 2006). Subsequent computational analysis of such datasets has confirmed that presented peptides are more likely to have a high binding affinity with MHC and are generally derived from high-abundance proteins (Johnson et al., 2009; Ben Dror et al., 2010; Hoof et al., 2012). Protein identification and abundance in skin has been addressed in recent proteomic studies and computational models are available for predicting the binding affinity of peptides with various HLA types (Vita et al., 2010; Gill et al., 2011; van Eijl et al., 2012). Although this ability to predict presented peptides exists, significant progress in this area, both experimentally and computationally, will be required in order to make predictions on modified pMHC molecules. For this aspect of the response, a practical first step would be to assume that the amount of pMHC per APC or the fraction of APCs able to induce a $\mathrm{T}$ cell response is proportional to bulk concentration of modified protein in skin.

A number of characteristics of the clonally expanded $\mathrm{T}$ cell population have been hypothesized as contributory factors in the establishment of ACD: breadth (the clonal diversity of the $\mathrm{T}$ cell response), quality (the balance achieved between effector and regulatory cells) and magnitude (the vigor and duration of proliferation and the clonal expansion of allergen-reactive $\mathrm{T}$ cells). The combined state of these three variables with respect to the sensitizing ingredient is thought to determine whether an individual will display the adverse response, i.e., has the potential to elicit an allergic contact dermatitis response (Kimber et al., 2012) (see "Organ/System Response" in Fig. 5). Of the three $\mathrm{T}$ cell response metrics that likely characterize the adverse response, predicting the magnitude of the $\mathrm{T}$ cell population is likely to be the most straightforward starting point for development of a toxicodynamic model; descriptions of how this population is partitioned into T cell subtypes (quality) or clonotypes (breadth) is a refinement that can be built upon the magnitude prediction as knowledge is acquired. While both $\mathrm{CD}^{+}$and $\mathrm{CD}^{+} \mathrm{T}$ cells are known to be involved, $\mathrm{CD}^{+} \mathrm{T}$ cells have been identified as the primary effector cells in the skin sensitization response (Kimber and Dearman, 2002). Thus the magnitude of the antigen-specific memory $\mathrm{CD}^{+} \mathrm{T}$ cell population may be considered a practical starting toxicodynamic metric with which to distinguish an adverse response from normal function. Very little sensitizer specific-data is available to build an understanding of how these three variables characterize the adverse response. However, more is known about the T cell proliferation dynamics in response to pathogens, and these data could serve 


$\begin{gathered}\text { Molecular } \\ \text { Initiating Event }\end{gathered}$
$\begin{gathered}\text { Cellular } \\ \text { Response }\end{gathered}$

Fig. 5: Relating the extent of the molecular initiating event to the likelihood or severity of the adverse response as defined by the AOP for skin sensitization

The key metrics (variables) of the sensitization response across levels of biological organization: from the molecular initiating event to the organism response. The key "Toxicodynamic Events of the AOP" are explicitly considered in relating the TD model input, concentration of modified protein $(\mathrm{mM})$, to the output, number of memory T cells. Quantifying these relationships in a TD model provides the relationship between the extent of molecular initiating event and the likelihood of an adverse response.

as a model response with which to populate a toxicodynamic model for skin sensitization.

For more than a decade, mechanism-based mathematical approaches have proved useful for understanding the viral dynamics of immune responses (Perelson and Weisbuch, 1997; Asquith and Bangham, 2003). Much of the research has focused on chronic infectious diseases such as human immunodeficiency virus (HIV), human T cell lymphotropic virus (HTLV), and hepatitis (Perelson, 2002; Wodarz, 2006; Asquith and Bangham, 2007). Acute viral infections also have been modeled, such as influenza and lymphocytic choriomeningitis virus (LCMV) in mice, with models that include mechanisms for activation and proliferation of naïve $\mathrm{T}$ cells (Bocharov, 1998; Bocharov et al., 2003; Tridane and Kuang, 2010) that provide a closer analog to the induction of skin sensitization. The predictions from these models have been successfully used to test hypotheses such as programmed $\mathrm{T}$ cell proliferation and to build an understanding of the dynamics of $\mathrm{T}$ cell proliferation (Kaech and Ahmed, 2001; Antia et al., 2003; Wherry et al., 2003). Modeling approaches such as stochastic modeling and agent-based modeling also have been used to describe variability in the dynamic response (Chao et al., 2004). Stochastic models capture probabilistic elements of the system, for example the probability of encounters between APCs and T cells in the lymph nodes, or variations in the response due to potentially small numbers of antigen-specific T cells. Agent-based models are even more detailed, directly simulating the behavior of individual agents (e.g., T cells) based on defined rules for their behavior and interactions.

A toxicodynamic model of the sensitizer-specific $\mathrm{CD} 8^{+} \mathrm{T}$ cell response would have to consider a number of tissues, immigrant APC populations, T cell subsets, and population dynamic processes. A pragmatic model of the $\mathrm{T}$ cell response should represent only cell types that are relevant to generation of the $\mathrm{CD}^{+} \mathrm{T}$ cell memory population (Fig. 6). This includes naïve $\mathrm{CD}^{+} \mathrm{T}$ cells $(\mathrm{N})$ as precursor cells, effector $\mathrm{CD}^{+} \mathrm{T}$ cells $(\mathrm{E})$ which have been shown to be involved in response to chemical allergens (Xu et al., 1996; Martin et al., 2000; Wang et al., 2000), and the critical memory populations. It is not known which memory population contributes to the acquisition of sensitization, so both the effector memory (EM) and central memory (CM) subtypes should be considered. The route of differentiation from naïve to memory and effector cells currently is hypothesized to occur by one of six distinct mechanisms (Ahmed et al., 2009; Kaech and Cui, 2012). Until further research uncovers which hypothesis is most likely, the key activated-naïve/precursor-memory state could be represented as an intermediate population (termed proliferating memory, PM). Following expansion in the lymph node, the T cells enter the blood and effector and effector memory $\mathrm{T}$ cells home to the skin. Explicitly modeling these $\mathrm{CD}^{+} \mathrm{T}$ cell populations in the blood and skin allows model predictions to be made that could be reconciled with clinical measurements in blood or from skin biopsies. The key parameters required for such a model would 


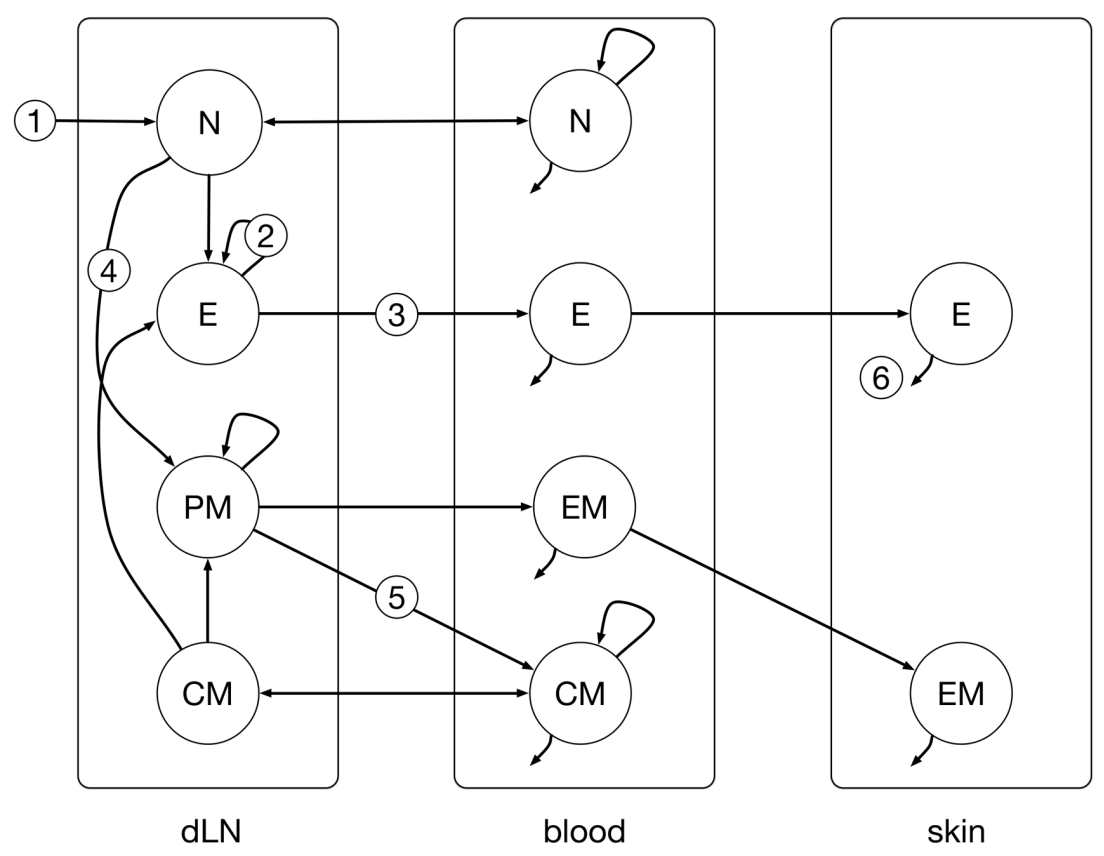

Fig. 6: Example schematic of a mechanistically-based toxicodynamic model of sensitizer induced CD8 ${ }^{+}$memory T cell population

Cell types that would likely be considered in a mechanistically-based toxicodynamic model of sensitizer induced CD8 ${ }^{+}$memory $\mathrm{T}$ cell population: $\mathrm{N}$ ) naïve $\mathrm{CD}^{+} \mathrm{T}$ cells; E) effector $\mathrm{CD} 8^{+} \mathrm{T}$ cells; $\mathrm{CM}$ ) central memory $\mathrm{CD}^{+} \mathrm{T}$ cells; EM) effector memory CD8 ${ }^{+} \mathrm{T}$ cells $\mathrm{PM}$ ) proliferating memory $\mathrm{CD}^{+} \mathrm{T}$ cells. Processes that would likely be represented: 1 ) migration of epidermal Langerhans cells and activation of naïve CD8 ${ }^{+} \mathrm{T}$ cells; 2 ) cell proliferation in the presence (lymph node) and absence (blood) of antigen; 3 ) cell trafficking between lymph node, blood, and skin; 4) differentiation to various $\mathrm{CD}^{+} \mathrm{T}$ cell subtypes; 5) cell trafficking and differentiation; 6) cell death.

be the death and proliferation rates of all cell types in both the absence and presence of antigen, the initial size of the naïve antigen-specific $\mathrm{CD} 8^{+} \mathrm{T}$ cell population available for clonal expansion, and the migration rates of cells between lymph node, blood and skin (see Fig. 6). Human data is available for many of these parameters based on measurements taken in blood in the absence of antigen (Mclean and Michie, 1995; Murray et al., 2003; Vrisekoop et al., 2008). However, in the presence of sensitizer stimulation, model parameterization becomes more challenging and may be built using information from other immune responses under the assumption that the underlying cell response mechanisms are common. One possible source of information is responses to viral infection (Ahmed and Akondy, 2011), although the most detailed quantitative data available in this case are from mice (Yoon et al., 2010, 2012). However, it may be that immune responses to passive antigens give a more relevant comparison to the case of sensitizers than responses to highly dynamic pathogens such as viruses. Examples of passive antigens include vaccines intended to produce a $\mathrm{CD} 8^{+}$response (Butler et al., 2011) or drugs that cause hypersensitivity (Ostrov et al., 2012).
The models of $\mathrm{T}$ cell dynamics discussed and their biological parameterizations may therefore serve as a good first approximation for a toxicodynamic model of $\mathrm{T}$ cell proliferation in response to sensitizing ingredients (see Fig. 4C). Translation of these models for skin sensitization responses and determination of the required model parameters, such as rates of antigenspecific proliferation, constitutes a significant experimental and clinical challenge (Kimber et al., 2012).

\section{Discussion}

We propose an approach to risk assessment of skin sensitization without animal testing that uses tailored data to support predictive physiologically- and mechanistically-based toxicokinetic-toxicodynamic modeling. Research into the feasibility of constructing such models and to provide a prediction of the $\mathrm{T}$ cell response for a given consumer exposure to specific sensitizing chemicals is underway (see Fig. 4). However, in order to use such models two questions must be answered: First, what confidence do we have in the model prediction of the $\mathrm{T}$ 
cell response? Second, is the predicted response indicative of an adverse sensitization response?

AOPs detail the known molecular, biochemical, cellular, organ- and organism-level effects of a toxicant on the biological system required for mechanism-based toxicodynamic modeling and development of the required supporting experimental approaches. Confidence in such mechanism-based models comes, first, from conviction in both the underlying mechanisms being truly causal events in the response and in the relevance of the experimental data used to inform those mechanisms and subsequent model parameterization. Conviction in the underlying mechanisms and data comes from continued scientific enquiry and robust peer review. Maintaining and updating a knowledge base for AOPs based on available scientific research should be a continual process and can be achieved in a collaborative manner via open-source tools such as http:// www.effectopedia.org. In addition to capturing and documenting the biological mechanisms, the model assumptions, structure, and parameterization must also be made transparent. For complex models with many parameters this in itself can be a significant challenge, but frameworks are available (Andrews et al., 2010). Furthermore, given the limitations, particularly in quantitative knowledge, TKTD modeling frameworks that explicitly capture uncertainty in underlying modeled data, parameters, and mechanisms would likely need to be employed (Alden et al., 2013). Ultimately, peer-review of AOPs in the scientific literature and discussion at a global regulatory level provides confidence and contributes to their acceptance for use in risk assessment (OECD, 2011, 2012a,b).

In addition to having an accepted mechanistic rationale, confidence in mechanistic TKTD models for risk assessment comes from to an ability to demonstrate their qualitative and quantitative predictive capability. This is a process of challenging the model with previously unseen observational data and determining the extent of the discrepancy between the observation and model prediction. In the context of the practical TKTD models discussed above for skin sensitization, this would amount to comparison of prediction and observation of the memory $\mathrm{T}$ cell population following sensitizer exposure (see Fig. 4). The observational data could be obtained using in vitro systems and compared to a TKTD model with an in vitro parameterization. Demonstrated in vitro predictive power would provide more confidence when changing to an in vivo parameterization and predicting the response to an actual exposure scenario in humans. Ideally, model predictions and observations should be made in the system of interest and, to this end, topical immunotherapy may provide an opportunity to ethically study the emergence of a sensitizer-specific $\mathrm{T}$ cell response in humans (Singh and Lavanya, 2010). The purpose of such an evaluation would be to provide sufficient confidence in the underlying mechanistic assumptions modeled in order to make predictions on novel ingredients for which no clinical data is available.

Understanding what constitutes an adverse outcome in terms of the underlying $\mathrm{T}$ cell response requires characterization of this response in subjects who have been diag- nosed "patch test positive" with contact allergy (Nedorost and Cooper, 2001; White, 2012). As discussed above, a number of characteristics of the $\mathrm{T}$ cell population have been hypothesized as contributory factors in the establishment of ACD including, breadth (the clonal diversity of the T cell response), quality (the balance achieved between effector and regulatory cells), and magnitude (the vigor and duration of proliferation and the clonal expansion of allergen-reactive $\mathrm{T}$ cells). The sensitization response generally is regarded to be a threshold response, i.e., there is an exposure threshold $(\mu \mathrm{g} /$ $\mathrm{cm}^{2}$ ) below which sensitization either does not occur or is not observed clinically (Kimber et al., 1999). This suggests that there exists a dose thresholded event in the biology beyond which the sensitization response emerges. Such a threshold could be either quantitative, e.g., the magnitude of a population of memory T cells below which sensitization is clinically silent, or qualitative, e.g., the population balance of $\mathrm{T}$ cells of a particular breadth of phenotype that correspond with sensitization. We propose the magnitude of the antigen-specific memory $\mathrm{CD} 8^{+} \mathrm{T}$ cell population as a reasonable starting metric with which to attempt to identify the adverse response threshold (see Fig. 4C). A qualitative and quantitative investigation of $\mathrm{T}$ cell populations and surrogate biomarkers in existing patient populations of sensitized individuals could be used to characterize what constitutes, clinically, an adverse $\mathrm{T}$ cell response. A particular challenge in this regard would be building an understanding of what aspects of the biology drive variability and incorporating this into the TKTD models.

Consumer safety risk assessment for skin sensitization has the objective of determining a safe exposure for product ingredients commensurate with the level of skin sensitization hazard (potency) they pose. The current paradigm is one of comparing consumer exposure with potency data and applying uncertainty factors for which there is historical precedent (Felter et al., 2002). Historically, such potency data has been generated in animals and equivalent prediction by non-animal methods is not currently regarded as possible (Adler et al., 2011). When the risk assessment is required to be performed without animal testing, then fully utilizing and quantifying the mechanistic knowledge on the adverse response is key. Adverse outcome pathways are a tool to capture current knowledge on the molecular, biochemical, cellular, organ- and organism-level effects of a toxicant on the biological system. Quantifying these effects in the context of consumer exposure could be achieved by constructing physiologically- and mechanistically-based TKTD models informed by tailored non-animal experimental data and theoretical chemistry predictions. Confidence in such an approach to skin allergy risk assessment could be achieved by better characterization of the sensitizer specific $\mathrm{T}$ cell response in humans and comparison of model predictions with clinical exposures known to result in the acquisition of ACD. Ultimately, such an approach will serve to put skin sensitization risk assessment on a sound theoretical, experimental, and clinical footing, robust and adaptable to the new discoveries and advances in the field of immunobiology. 


\section{References}

Adler, S., Basketter, D., Creton, S., et al. (2011). Alternative (non-animal) methods for cosmetics testing: current status and future prospects - 2010. Arch Toxicol 85, 367-485.

Ahmed, R., Bevan, M. J., Reiner, S. L., et al. (2009). The precursors of memory: models and controversies. Nat Rev Immunol 9, 662-668.

Ahmed, R. and Akondy, R. S. (2011). Insights into human CD8(+) T-cell memory using the yellow fever and smallpox vaccines. Immunol Cell Biol 89, 340-345.

Alden, K., Read, M., Timmis, J., et al. (2013). Spartan: A comprehensive tool for understanding uncertainty in simulations of biological systems. PLoS Comput Biol 9, e1002916.

Andersen, M. E. (2003). Toxicokinetic modeling and its applications in chemical risk assessment. Toxicol Lett 138, 9-27.

Andrews, P. S., Polack, F. A. C., Sampson, A.T., et al. (2010). The CoSMoS process, version 0.1: a process for the modelling and simulation of complex systems. University of York Technical Report YCS - 2010, 453.

Ankley, G. T., Bennett, R. S., Erickson, R. J., et al. (2010). Adverse outcome pathways: a conceptual framework to support ecotoxicology research and risk assessment. Environ Toxicol Chem 29, 730-741.

Antia, R., Bergstrom, C. T., Pilyugin, S. S., et al. (2003). Models of CD8+ responses: 1 . What is the antigen-independent proliferation program. $J$ Theor Biol 221, 585-598.

Api, A. M., Basketter, D. A., Cadby, P. A., et al. (2008). Dermal sensitization quantitative risk assessment (QRA) for fragrance ingredients. Regul Toxicol Pharmacol 52, 3-23.

Ashauer, R., Agatz, A., Albert, C., et al. (2011). Toxicokinetictoxicodynamic modeling of quantal and graded sublethal endpoints: a brief discussion of concepts. Environ Toxicol Chem 30, 2519-2524.

Asquith, B. and Bangham, C. R. M. (2003). An introduction to lymphocyte and viral dynamics: the power and limitations of mathematical analysis. Proc R Soc Lond B Biol Sci 270, 1651-1657.

Asquith, B. and Bangham, C. R. M. (2007). Quantifying HTLV-I dynamics. Immunol Cell Biol 85, 280-286.

Basketter, D., Dooms-Goossens, A., Karlberg, A. T., et al. (1995). The chemistry of contact allergy: why is a molecule allergenic? Contact Dermatitis 32, 65-73.

Basketter, D. A., Evans, P., Fielder, R. J., et al. (2002). Local lymph node assay - validation, conduct and use in practice. Food Chem Toxicol 40, 593-598.

Basketter, D., Pease, C., Kasting, G., et al. (2007). Skin sensitisation and epidermal disposition: the relevance of epidermal disposition for sensitisation hazard identification and risk assessment. The report and recommendations of ECVAM workshop 59. Altern Lab Anim 35, 137-154.

Basketter, D. A. and Kimber, I. (2010). Contact Hypersensitivity (397-411). In C. A. McQueen (ed.), Comprehensive Toxicology. Elsevier.

Bauch, C., Kolle, S. N., Ramirez, T., et al. (2012). Putting the parts together: combining in vitro methods to test for skin sensitizing potentials. Regul Toxicol Pharmacol 63, 489-504.
Bauer, B., Andersson, S. I., Stenfeldt, A.-L., et al. (2011). Modification and expulsion of keratins by human epidermal keratinocytes upon hapten exposure in vitro. Chem Res Toxicol 24, 737-743.

Ben Dror, L., Barnea, E., Beer, I., et al. (2010). The HLA-B*2705 peptidome. Arthritis Rheum 62, 420-429.

Bhattacharya, S., Conolly, R. B., Kaminski, N. E., et al. (2010). A bistable switch underlying B-cell differentiation and its disruption by the environmental contaminant 2,3,7,8-tetrachlorodibenzo-p-dioxin. Toxicol Sci 115, 51-65.

Bhattacharya, S., Zhang, Q., Carmichael, P. L., et al. (2011). Toxicity testing in the 21 century: defining new risk assessment approaches based on perturbation of intracellular toxicity pathways. PloS One 6, e20887.

Bocharov, G. A. (1998). Modelling the dynamics of LCMV infection in mice: conventional and exhaustive CTL responses. J Theor Biol 192, 283-308.

Bocharov, G., Klenerman, P., and Ehl, S. (2003). Modelling the dynamics of LCMV infection in mice: II. Compartmental structure and immunopathology. J Theor Biol 221, 349-378.

Böhme, A., Thaens, D., Paschke, A., et al. (2009). Kinetic glutathione chemoassay to quantify thiol reactivity of organic electrophiles - application to alpha,beta-unsaturated ketones, acrylates, and propiolates. Chem Res Toxicol 22, 742-750.

Bonnist, E. Y. M., Gorce, J.-P., Mackay, C., et al. (2011). Measuring the penetration of a skin sensitizer and its delivery vehicles simultaneously with confocal Raman spectroscopy. Skin Pharmacol Physiol 24, 274-283.

Boroujerdi, M. (2001). Pharmacokinetics: Principles and Applications. New York, USA: McGraw-Hill Inc.

Butler, N. S., Nolz, J. C., and Harty, J. T. (2011). Immunologic considerations for generating memory CD8 T cells through vaccination. Cell Microbiol 13, 925-933.

Carreño, L. J., González, P. A., and Kalergis, A. M. (2006). Modulation of $\mathrm{T}$ cell function by TCR/pMHC binding kinetics. Immunobiology 211, 47-64.

Chao, D. L., Davenport, M. P., Forrest, S., et al. (2004). A stochastic model of cytotoxic T cell responses. J Theor Biol 228, 227-240.

Chipinda, I., Ajibola, R. O., Morakinyo, M. K., et al. (2010). Rapid and simple kinetics screening assay for electrophilic dermal sensitizers using nitrobenzenethiol. Chem Res Toxicol 23, 918-925.

Christensson, J. B., Matura, M., Gruvberger, B., et al. (2010). Linalool - a significant contact sensitizer after air exposure. Contact Dermatitis 62, 32-41.

Dancik, Y., Miller, M. A., Jaworska, J., et al. (2013). Design and performance of a spreadsheet-based model for estimating bioavailability of chemicals from dermal exposure. Adv Drug Delivery Rev 65, 221-236.

Davies, M., Pendlington, R. U., Page, L., et al. (2011). Determining epidermal disposition kinetics for use in an integrated nonanimal approach to skin sensitization risk assessment. Toxicol Sci 119, 308-318.

Felter, S. P., Robinson, M. K., Basketter, D. A., et al. (2002). A review of the scientific basis for uncertainty factors for use 
in quantitative risk assessment for the induction of allergic contact dermatitis. Contact Dermatitis 47, 257-266.

Felter, S. P., Ryan, C. A., Basketter, D. A., et al. (2003). Application of the risk assessment paradigm to the induction of allergic contact dermatitis. Regul Toxicol Pharmacol 37, 1-10.

Gebreselassie, D., Spiegel, H., and Vukmanovic, S. (2006). Sampling of major histocompatibility complex class I-associated peptidome suggests relatively looser global association of HLA-B*5101 with peptides. Hum Immunol 67, 894-906.

Gibbs, S., van de Sandt, J. J. M., Merk, H. F., et al. (2007). Xenobiotic metabolism in human skin and 3D human skin reconstructs: a review. Curr Drug Metab 8, 758-772.

Gill, C., Parkinson, E., Church, M. K., et al. (2011). A qualitative and quantitative proteomic study of human microdialysate and the cutaneous response to injury. AAPS J 13, 309-317.

Götz, C., Pfeiffer, R., Tigges, J., et al. (2012). Xenobiotic metabolism capacities of human skin in comparison with a 3Depidermis model and keratinocyte-based cell culture as in vitro alternatives for chemical testing: phase II enzymes. Exp Dermatol 21, 364-369.

Hagvall, L., Baron, J. M., Börje, A., et al. (2008). Cytochrome P450-mediated activation of the fragrance compound geraniol forms potent contact allergens. Toxicol Appl Pharmacol 233, 308-313.

Herkenne, C., Alberti, I., Naik, A., et al. (2008). In vivo methods for the assessment of topical drug bioavailability. Pharm Res 25, 87-103.

Hickman, H. D., Luis, A. D., Buchli, R., et al. (2004). Toward a definition of self: proteomic evaluation of the class I peptide repertoire. J Immunol 172, 2944-2952.

Holmgaard, R., Nielsen, J. B., and Benfeldt, E. (2010). Microdialysis sampling for investigations of bioavailability and bioequivalence of topically administered drugs: current state and future perspectives. Skin Pharmacol Physiol 23, 225243.

Hoof, I., van Baarle, D., Hildebrand, W. H., et al. (2012). Proteome sampling by the HLA class I antigen processing pathway. PLoS Comput Biol 8, e1002517.

Ibrahim, R., Nitsche, J. M., and Kasting, G. B. (2012). Dermal clearance model for epidermal bioavailability calculations. $J$ Pharm Sci 101, 2094-2108.

Jacques, C., Perdu, E., Duplan, H., et al. (2010). Disposition and biotransformation of 14C-Benzo(a)pyrene in a pig ear skin model: ex vivo and in vitro approaches. Toxicol Lett 199, 2233.

Jaworska, J., Harol, A., Kern, P. S., et al. (2011). Integrating non-animal test information into an adaptive testing strategy - skin sensitization proof of concept case. ALTEX 28, 211225 .

Jaworska, J., Dancik, Y., Kern, P., et al. (2013). Bayesian integrated testing strategy to assess skin sensitization potency: from theory to practice. J Appl Toxicol May 14. doi: 10.1002/ jat.2869 (Epub ahead of print).

Johnson, K. L., Ovsyannikova, I. G., Mason, C. J., et al. (2009). Discovery of naturally processed and HLA-presented class I peptides from vaccinia virus infection using mass spectrometry for vaccine development. Vaccine 28, 38-47.

Jowsey, I. R., Basketter, D. A., Westmoreland, C., et al. (2006). A future approach to measuring relative skin sensitising potency: a proposal. J Appl Toxicol 26, 341-350.

Judson, R. S., Kavlock, R. J., Setzer, R. W., et al. (2011). Estimating toxicity-related biological pathway altering doses for high-throughput chemical risk assessment. Chem Res Toxicol 24, 451-462.

Kaech, S. M. and Ahmed, R. (2001). Memory CD8 ${ }^{+}$T cell differentiation: initial antigen encounter triggers a developmental program in naïve cells. Nat Immunol 2, 415-422.

Kaech, S. M. and Cui, W. (2012). Transcriptional control of effector and memory $\mathrm{CD}^{+} \mathrm{T}$ cell differentiation. Nat Rev Immunol 12, 749-761.

Karlberg, A.-T., Bergström, M. A., Börje, A., et al. (2008). Allergic contact dermatitis - formation, structural requirements, and reactivity of skin sensitizers. Chem Res Toxicol 21, 53-69.

Kimber, I., Gerberick, G. F., and Basketter, D. A. (1999). Thresholds in contact sensitization: theoretical and practical considerations. Food Chem Toxicol 37, 553-560.

Kimber, I. and Dearman, R. J. (2002). Allergic contact dermatitis: the cellular effectors. Contact Dermatitis 46, 1-5.

Kimber, I. and Dearman, R. J. (2003). What makes a chemical an allergen? Ann Allergy Asthma Immunol 90, 28-31.

Kimber, I., Basketter, D. A., Gerberick, G. F., et al. (2011). Chemical allergy: translating biology into hazard characterization. Toxicol Sci 120, Suppl, S238-268.

Kimber, I., Maxwell, G., Gilmour, N., et al. (2012). Allergic contact dermatitis: a commentary on the relationship between T lymphocytes and skin sensitising potency. Toxicology 291, 18-24.

Krewski, D., Acosta, D., Andersen, M., et al. (2010). Toxicity testing in the $21^{\text {st }}$ century: a vision and a strategy. $J$ Toxicol Environ Health Part B Crit Rev 13, 51-138.

Landsteiner, K. and Di Somma, A. A. (1938). Studies on the sensitization of animals with simple chemical compounds : V. sensitisation to diazomethane and mustard oil. J Exp Med 68, 505-512.

Lau, C., Andersen, M. E., Crawford-Brown, D. J., et al. (2000). Evaluation of biologically based dose-response modeling for developmental toxicity: a workshop report. Regul Toxicol Pharmacol 31, 190-199.

Lepoittevin, J. P., Basketter, D. A., Goossens, A., et al. (eds.) (2011). Allergic Contact Dermatitis: The Molecular Basis. Berlin, Germany: Springer.

Louisse, J., de Jong, E., van de Sandt, J. J. M., et al. (2010). The use of in vitro toxicity data and physiologically based kinetic modeling to predict dose-response curves for in vivo developmental toxicity of glycol ethers in rat and man. Toxicol Sci $118,470-484$.

Magnusson, B. M., Anissimov, Y. G., Cross, S. E., et al. (2004). Molecular size as the main determinant of solute maximum flux across the skin. J Invest Dermatol 122, 993-999.

Martin, S. and Weltzien, H. U. (1994). T cell recognition of 
haptens, a molecular view. Int Arch Allergy Immunol 104, 10-16.

Martin, S., Lappin, M. B., Kohler, J., et al. (2000). Peptide immunization indicates that CD8+ T cells are the dominant effector cells in trinitrophenyl-specific contact hypersensitivity. J Invest Dermatol 115, 260-266.

Maxwell, G. and Mackay, C. (2008). Application of a systems biology approach to skin allergy risk assessment. Altern Lab Anim 36, 521-556.

McCarley, K. D. and Bunge, A. L. (2001). Pharmacokinetic models of dermal absorption. J Pharm Sci 90, 1699-1719.

Mclean, A. R. and Michie, C. A. (1995). In vivo estimates of division and death rates of human T lymphocytes. Proc Natl Acad Sci U S A 92, 3707-3711.

Mitragotri, S. (2003). Modeling skin permeability to hydrophilic and hydrophobic solutes based on four permeation pathways. J Controlled Release 86, 69-92.

Mitragotri, S., Anissimov, Y. G., Bunge, A. L., et al. (2011). Mathematical models of skin permeability: an overview. Int $J$ Pharm 418, 115-129.

Morris, G. P. and Allen, P. M. (2012). How the TCR balances sensitivity and specificity for the recognition of self and pathogens. Nat Immunol 13, 121-128.

Murray, J. M., Kaufmann, G. R., Hodgkin, P. D., et al. (2003). Naive $\mathrm{T}$ cells are maintained by thymic output in early ages but by proliferation without phenotypic change after age twenty. Immunol Cell Biol 81, 487-495.

Nedorost, S. T. and Cooper, K. D. (2001). The role of patch testing for chemical and protein allergens in atopic dermatitis. Curr Allergy Asthma Rep 1, 323-328.

Notman, R. and Anwar, J. (2013). Breaching the skin barrier insights from molecular simulation of model membranes. $A d v$ Drug Delivery Rev 65, 237-250.

OECD (2011). Report of the workshop on using mechanistic information in forming chemical categories. OECD Environment, Health and Safety Publications Series on Testing and Assessment 138, 1-176.

OECD (2012a). The Adverse Outcome Pathway for Skin Sensitisation Initiated by Covalent Binding to Proteins. Part 1: Scientific Edvidence. OECD Environment, Health and Safety Publications Series on Testing and Assessment 168, 1-59.

OECD (2012b). The Adverse Outcome Pathway for Skin Sensitisation Initiated by Covalent Binding to Proteins. Part 2: Use of the AOP to Develop Chemical Categories and Integrated Assessment and Testing Approaches. OECD Environment, Health and Safety Publications Series on Testing and Assessment 168, 1-46.

Ostrov, D. A., Grant, B. J., Pompeu, Y. A., et al. (2012). Drug hypersensitivity caused by alteration of the MHC-presented self-peptide repertoire. Proc Natl Acad Sci U S A 109, 99599964.

Otto, A., du Plessis, J., and Wiechers, J. W. (2009). Formulation effects of topical emulsions on transdermal and dermal delivery. Int J Cosmet Sci 31, 1-19.

Pendlington, R. U., Minter, H. J., Stupart, L., et al. (2008). De- velopment of a modified in vitro skin absorption method to study the epidermal/dermal disposition of a contact allergen in human skin. Cutan Ocul Toxicol 27, 283-294.

Perelson, A. and Weisbuch, G. (1997). Immunology for physicists. Rev Mod Phys 69, 1219-1268.

Perelson, A. S. (2002). Modelling viral and immune system dynamics. Nat Rev Immunol 2, 28-36.

Pickard, C., Louafi, F., McGuire, C., et al. (2009). The cutaneous biochemical redox barrier: a component of the innate immune defenses against sensitization by highly reactive environmental xenobiotics. J Immunol 183, 7576-7584.

Potts, R. O. and Guy, R. H. (1992). Predicting skin permeability. Pharm Res 9, 663-669.

Reddy, M., Yang, R. S., Andersen, M. E., et al. (2005). Physiologically Based Pharmacokinetic Modeling : Science and Applications. Hoboken, USA: Wiley-Interscience.

Rim, J. E., Pinsky, P. M., and van Osdol, W. W. (2009). Multiscale modeling framework of transdermal drug delivery. Ann Biomed Eng 37, 1217-1229.

Roberts, D. W. and Natsch, A. (2009). High throughput kinetic profiling approach for covalent binding to peptides: application to skin sensitization potency of Michael acceptor electrophiles. Chem Res Toxicol 22, 592-603.

Rotroff, D. M., Wetmore, B. A., Dix, D. J., et al. (2010). Incorporating human dosimetry and exposure into high-throughput in vitro toxicity screening. Toxicol Sci 117, 348-358.

Shuey, D. L., Setzer, R. W., Lau, C., et al. (1995). Biological modeling of 5-fluorouracil developmental toxicity. Toxicology 102, 207-13.

Simonsson, C., Andersson, S. I., Stenfeldt, A.-L., et al. (2011). Caged fluorescent haptens reveal the generation of cryptic epitopes in allergic contact dermatitis. J Invest Dermatol 131, 1486-1493.

Singh, G. and Lavanya, M. (2010). Topical immunotherapy in alopecia areata. Int J Trichol 2, 36-39.

Stone, J. D., Chervin, A. S., and Kranz, D. M. (2009). T-cell receptor binding affinities and kinetics: impact on T-cell activity and specificity. Immunology 126, 165-176.

Thompson, C. M., Sonawane, B., Barton, H. A., et al. (2008). Approaches for applications of physiologically based pharmacokinetic models in risk assessment. J Toxicol Environ Health Part B Crit Rev 11, 519-547.

Tridane, A. and Kuang, Y. (2010). Modeling the interaction of cytotoxic T lymphocytes and influenza virus infected epithelial cells. Math Biosci Eng 7, 171-185.

USEPA (2005). Guidelines for carcinogen risk assessment. EPA/630/P-03/001F

Van Eijl, S., Zhu, Z., Cupitt, J., et al. (2012). Elucidation of xenobiotic metabolism pathways in human skin and human skin models by proteomic profiling. PloS One 7, e41721.

Vita, R., Zarebski, L., Greenbaum, J. A., et al. (2010). The immune epitope database 2.0. Nucleic Acids Res. 38, D854862 .

Vrisekoop, N., den Braber, I., de Boer, A. B., et al. (2008). Sparse production but preferential incorporation of recently 
produced naive T cells in the human peripheral pool. Proc Natl Acad Sci U S A 105, 6115-6120.

Wang, B., Fujisawa, H., Zhuang, L., et al. (2000). CD4 ${ }^{+}$Th1 and $\mathrm{CD}^{+}$type 1 cytotoxic $\mathrm{T}$ cells both play a crucial role in the full development of contact hypersensitivity. J Immunol 165, 6783-6790.

Wang, T.-F., Kasting, G. B., and Nitsche, J. M. (2006). A multiphase microscopic diffusion model for stratum corneum permeability. I. Formulation, solution, and illustrative results for representative compounds. J Pharm Sci 95, 620-648.

Wang, C. Y. and Maibach, H. I. (2011). Why minimally invasive skin sampling techniques? A bright scientific future. Cutan Ocul Toxicol 30, 1-6.

Watanabe, K. H., Andersen, M. E., Basu, N., et al. (2011). Defining and modeling known adverse outcome pathways: Domoic acid and neuronal signaling as a case study. Environ Toxicol Chem 30, 9-21.

Welling, P.G. (1997). Pharmacokinetics: Processes, Mathematics, and Applications. Washington, USA: American Chemical Society.

Weltzien, H. U., Moulon, C., Martin, S., et al. (1996). T cell immune responses to haptens. Structural models for allergic and autoimmune reactions. Toxicology 107, 141-151.

Wherry, E. J., Teichgräber, V., Becker, T. C., et al. (2003). Lineage relationship and protective immunity of memory CD8 T cell subsets. Nat Immunol 4, 225-234.

White, J. M. L. (2012). Patch testing: what allergists should know. Clin Exp Allergy 42, 180-185.

Wodarz, D. (2006). Killer Cell Dynamics: Mathematical and Computational Approaches to Immunology (Interdisciplinary Applied Mathematics). New York, USA: Springer.

Xu, H., DiIulio, N. A., and Fairchild, R. L. (1996). T cell populations primed by hapten sensitization in contact sensitivity are distinguished by polarized patterns of cytokine production: interferon gamma-producing (Tc1) effector $\mathrm{CD} 8^{+} \mathrm{T}$ cells and interleukin (Il) 4/Il-10-producing (Th2) negative regulator. J Exp Med 183, 1001-1012.

Yoon, H., Kim, T. S., and Braciale, T. J. (2010). The cell cycle time of $\mathrm{CD}^{+} \mathrm{T}$ cells responding in vivo is controlled by the type of antigenic stimulus. PloS One 5, e15423.

Yoon, M., Campbell, J. L., Andersen, M. E., et al. (2012). Quantitative in vitro to in vivo extrapolation of cell-based toxicity assay results. Crit Rev Toxicol 42, 633-652.

Zalko, D., Ja cques, C., Duplan, H., et al. (2011). Viable skin efficiently absorbs and metabolizes bisphenol A. Chemosphere $82,424-430$.

\section{Acknowledgements}

This research is part of Unilever's ongoing effort to develop novel ways of delivering consumer safety. We would like to thank the many Unilever scientists and collaborators for their contributions.

\section{Correspondence to}

Cameron MacKay, PhD

Safety \& Environmental Assurance Centre

Unilever

Colworth Science Park

Sharnbrook

Bedford

MK44 1LQ, UK

Phone: +44 1234264924

e-mail: cameron.mackay@unilever.com 